\title{
On the continuity of multivariate Lagrange interpolation at natural lattices
}

\author{
J.-P. Calvi and V. M. Phung
}

\begin{abstract}
We give a natural geometric condition that ensures that sequences of interpolation polynomials (of fixed degree) of sufficiently differentiable functions with respect to the natural lattices introduced by Chung and Yao converge to a Taylor polynomial.
\end{abstract}

\section{Introduction}

\subsection{Stating the problem}

When $d+1$ points $a_{0}, \ldots, a_{d}$ in $\mathbb{R}$ converge to a limit point $a$, the corresponding Lagrange interpolation polynomial $\mathbf{L}\left[a_{0}, \ldots, a_{d} ; f\right]$ of a function $f$ at the points $a_{i}$ tends to the Taylor polynomial of $f$ at $a$ to the order $d$ and this under the sole assumption that $f$ is $d$ times continuously differentiable on a neighbourhood of the limit point. This classical result is an easy consequence of Newton's formula for Lagrange interpolation and of the mean value theorem for divided differences. In this paper, we study a multivariate analogue of this problem. We suppose that the points of a multivariate interpolation lattice $A$ of degree $d$ in $\mathbb{R}^{N}$ converge to a limit point $a \in \mathbb{R}^{N}$ and ask under what conditions we can assert that the corresponding multivariate Lagrange interpolation polynomials of a function $f$ converge to the Taylor polynomial of $f$ at $a$ to the order $d$ ? The question is answered for a particular but important class of interpolation lattices, the so-called natural lattices, see below.

\subsection{A known criterion}

In the multivariate case, a simple clear-cut answer for the above question cannot be expected. We recall a rather general criterion (which actually works for Hermitian interpolations) which can be found in [1]. Let us mention that the first results which appeared in the literature concerned the case (of practical importance in finite elements theory) for which the lattices are of the form $A^{(t)}=U^{(t)}(A)$ where $U^{(t)}$ is a sequence of linear transformations whose norms tend to 0 and $A$ is a fixed lattice. We refer to [1] for details and references to earlier works. We mention that the general problem of finding the possible limits for sequences of Lagrange interpolation polynomials was investigated in several recent papers, see for example $[\mathbf{5 , 1 0 , 1 1}$.

We denote by $\mathcal{P}^{d}\left(\mathbb{R}^{N}\right)$ the space of polynomials in $N$ real variables of degree at most $d$, $X^{\alpha}$ is the monomial function corresponding to the $N$-index $\alpha$, that is $X^{\alpha}(x)=x_{1}^{\alpha_{1}} \ldots x_{N}^{\alpha_{N}}$ for $x=\left(x_{1}, \ldots, x_{N}\right) \in \mathbb{R}^{N}$. The length of $\alpha$ is the degree of $X^{\alpha},|\alpha|=\sum_{i=1}^{N} \alpha_{i}$. We denote by $m_{d}$ the dimension of the vector space $\mathcal{P}^{d}\left(\mathbb{R}^{N}\right)$. We have $m_{d}=\left(\begin{array}{c}N+d \\ d\end{array}\right)$. In the whole paper, $N \geqslant 2$.

Theorem 1.1 (Bloom and Calvi). Let $A^{(s)}$ be a sequence of interpolation lattices of degree $d$ in $\mathbb{R}^{N}$. If the following condition holds

$$
|\alpha|=d+1 \Longrightarrow \lim _{s \rightarrow \infty} \mathbf{L}\left[A^{(s)} ; X^{\alpha}\right]=0
$$

Received 22 April 2012; revised 4 October 2012.

2010 Mathematics Subject Classification 41A05, 41A63, 41A80, 65D05 (primary). 
then, for every function $f$ of class $C^{m_{d}-1}$ in a neighbourhood of the origin 0 , we have

$$
\lim _{s \rightarrow \infty} \mathbf{L}\left[A^{(s)} ; f\right]=\mathbf{T}_{0}^{d}(f),
$$

where $\mathbf{L}\left[A^{(s)} ; \cdot\right]$ (respectively $\left.\mathbf{T}_{0}^{d}(\cdot)\right)$ denotes the Lagrange interpolation projector at the points of $A^{(s)}$ (respectively the Taylor projector at 0 of order $d$ ).

Unfortunately condition (1.1) is not easy to verify, especially if the degree of interpolation is not small, and it seems difficult to check it on general classes of interpolation lattices. Besides, Theorem 1.1 requires a high order of smoothness. We point out, however, that although it is not known whether the level of differentiability required in Theorem 1.1 is optimal (in the case of Lagrange interpolation), examples do exist for which convergence does not hold for functions of class $C^{d+1}$ but holds for functions of higher smoothness, see [1, Example 5.4].

The aim of this paper is to give a natural geometric condition in the case where the interpolation lattices are natural lattices. From an algebraic point of view, they can be regarded as the simplest interpolation lattices: every point is situated at the intersection of $N$ hyperplanes chosen among a minimal family and the corresponding Lagrange fundamental polynomials are products of affine forms. The definition and main properties of natural lattices are collected in $\S 2$. Our criterion is given and commented in $\S 3$. The proof is quite technical and is postponed to $\S 4$. It relies on a remainder formula due to Carl de Boor. The referee pointed out that the basic idea of using invariances in remainder formula was previously used in $[\mathbf{2}, \mathbf{6}]$, see also the survey paper [8].

We need very few facts from general interpolation theory. They are recalled in the following subsection.

\subsection{Basic facts on interpolation}

Let $E$ be a $m$-dimensional space of functions on $\mathbb{R}^{N}$ and $A=\left\{a_{1}, \ldots, a_{m}\right\} \subset \mathbb{R}^{N}$. We say that $A$ is an interpolation lattice for $E$ if for every function $f$ defined on $A$ there exists a unique $L \in E$ such that $L=f$ on $A$. Given a basis $\mathbf{f}=\left(f_{1}, \ldots, f_{m}\right)$ of $E$, we define the Vandermonde determinant $\operatorname{VDM}(\mathbf{f} ; A)$ by

$$
\operatorname{VDM}(\mathbf{f} ; A):=\operatorname{det}\left(f_{i}\left(a_{j}\right)\right)_{i, j=1}^{m} .
$$

Then $A$ is an interpolation lattice if and only if

$$
\operatorname{VDM}(\mathbf{f} ; A) \neq 0 \text {. }
$$

Of course, the condition is independent from the choice of the basis $\mathbf{f}$. When (1.4) is satisfied, we have

$$
L=\sum_{i=1}^{m} f\left(a_{i}\right) \mathbf{l}\left(A, a_{i}, \cdot\right),
$$

where $\mathbf{l}\left(A, a_{i}, \cdot\right)$ is the unique element of $E$ which vanishes on $A \backslash\left\{a_{i}\right\}$ and takes the value 1 at $a_{i}$,

$$
\mathbf{l}\left(A, a_{i}, x\right)=\frac{\operatorname{VDM}\left(\mathbf{f} ;\left\{a_{1}, \ldots, a_{i-1}, x, a_{i+1}, \ldots, a_{m}\right\}\right)}{\operatorname{VDM}(\mathbf{f} ; A)}, \quad 1 \leqslant i \leqslant m, x \in \mathbb{R}^{N} .
$$

In the case where $E=\mathcal{P}^{d}\left(\mathbb{R}^{N}\right)$ we write $L=\mathbf{L}[A ; f]$ and call it the Lagrange interpolation of $f$ at $A$. We say that $A$ is an interpolation lattice of degree $d$. The only other case that we consider in this paper is $E=\mathcal{H}^{d}\left(\mathbb{R}^{N}\right)$, the space of homogeneous polynomials of degree $d$ in $N$ variables whose dimension is $\left(\begin{array}{c}N+d-1 \\ d\end{array}\right)$.

Note that, as defined here, an interpolation lattice does not need to have an underlying affine structure. We shall however focus our attention on natural lattices, see below, which possess such underlying structure. 


\section{Natural lattices}

We recall the construction of the lattices and of some objects attached to them. Despite their apparent simplicity, it seems that these configurations were first considered in Chung and Yao's 1977 paper [3]. Here, we essentially follow the presentation and notational conventions of de Boor [4].

We work in $\mathbb{R}^{N}$ endowed with its canonical euclidean structure. The corresponding scalar product is denoted by $\langle\cdot, \cdot\rangle$.

A set of $N$ hyperplanes $H=\left\{\ell_{1}, \ldots, \ell_{N}\right\}$ in $\mathbb{R}^{N}$ is said to be in general position if the intersection of the $N$ hyperplanes is a singleton, that is

$$
\bigcap_{i=1}^{N} \ell_{i}=\left\{\vartheta_{H}\right\}
$$

If $\ell_{i}=\left\{x \in \mathbb{R}^{N}:\left\langle n_{i}, x\right\rangle=c_{i}\right\}, i=1, \ldots, N$, then $H$ is in general position if and only if $\operatorname{det}\left(n_{1}, \ldots, n_{N}\right) \neq 0$.

Definition 1. A collection $\mathbb{H}$ of (at least $N$ ) distinct hyperplanes in $\mathbb{R}^{N}$ is said to be in general position if:

(i) every $H \in\left(\begin{array}{l}\mathbb{H} \\ N\end{array}\right)$, that is, every subset of $N$ hyperplanes in $\mathbb{H}$, is in general position (as defined above);

(ii) the map

$$
H \in\left(\begin{array}{c}
\mathbb{H} \\
N
\end{array}\right) \longmapsto \vartheta_{H}:=\bigcap_{\ell \in H} \ell \in \mathbb{R}^{N}
$$

is one-to-one. Here and in the sequel we identify the singleton $\bigcap_{\ell \in H} \ell$ with its element.

As shown by the following theorem, the points obtained above as intersections of hyperplanes form an interpolation lattice of a certain degree.

Theorem 2.1 (Chung and Yao [3]). Let $\mathbb{H}$ be a set of $d \geqslant N$ hyperplanes in general position in $\mathbb{R}^{N}$. The lattice

$$
\Theta_{\mathbb{H}}=\left\{\vartheta_{H}=\bigcap_{\ell \in H} \ell: H \in\left(\begin{array}{c}
\mathbb{H} \\
N
\end{array}\right)\right\}
$$

is an interpolation lattice of degree $d-N$. Moreover, if $\ell \in \mathbb{H}$ is given by $\ell=\left\{x \in \mathbb{R}^{N}\right.$ : $\left.\left\langle n_{\ell}, x\right\rangle=c_{\ell}\right\}$ then we have the interpolation formula

$$
\mathbf{L}\left[\Theta_{\mathbb{H}} ; f\right](x)=\sum_{H \in\left(\begin{array}{c}
\mathbb{H} \\
N
\end{array}\right)} f\left(\vartheta_{H}\right) \prod_{\ell \notin H} \frac{\left\langle n_{\ell}, x\right\rangle-c_{\ell}}{\left\langle n_{\ell}, \vartheta_{H}\right\rangle-c_{\ell}} .
$$

The lattice $\Theta_{\mathbb{H}}$ is called a natural lattice (of degree $d-N$ ) and the interpolation formula is called the Chung-Yao interpolation formula corresponding to $\mathbb{H}$. In particular, we have

$$
\mathrm{l}\left(\Theta_{\mathbb{H}}, \vartheta_{H}, x\right)=\prod_{\ell \notin H} \frac{\left\langle n_{\ell}, x\right\rangle-c_{\ell}}{\left\langle n_{\ell}, \vartheta_{H}\right\rangle-c_{\ell}}, \quad H \in\left(\begin{array}{c}
\mathbb{H} \\
N
\end{array}\right) .
$$

When no confusion is possible, we omit the subscript $\mathbb{H}$ and write $\Theta$ instead of $\Theta_{\mathbb{H}}$. Of course, in (2.3), different equations for the hyperplanes yield the same formula. In the particular case $N=1$, every set of interpolation nodes may be regarded as a (trivial) natural lattice.

As shown by (2.3), interpolation polynomials at natural lattices are easy to compute. Some difficulties, however, must be pointed out. In constructing a natural lattice, we start from a family of hyperplanes and compute the interpolation points by solving, in principle, $m_{d}$ linear 
systems (of order $N$ ). Besides, it is a difficult problem, even in the case $N=2$, to decide how to choose the hyperplanes if a special requirement is made on the location of the interpolation points. For instance, we currently do not know what kind of limiting distribution we can obtain with a growing number of Chung-Yao points (that is, points of natural lattices). We mention that an interesting natural lattice was constructed by Sauer and $\mathrm{Xu}[\mathbf{9}]$ on bi-dimensional disks.

\section{Natural lattices of points converging to the origin}

\subsection{The convergence theorem}

From now on, we shall identify an hyperplane $\ell$ with the affine form $\ell(x)=\langle\mathbf{n}, x\rangle-c$ which defines it, where $\mathbf{n}$ is normalized so that $\|\mathbf{n}\|=1$. This abuse of language (each hyperplane has two normalized equations) should not create confusion. Boldfaced $\mathbf{n}$ will be kept for normalized vectors and vectors derived from them.

Supposing that the points of a sequence $\Theta^{(s)}$ of natural lattices of the same degree converge to the origin (or to any other fixed point), we study under what conditions the corresponding interpolation operator converges to the Taylor projector at the origin. Our main result is summarized in the following theorem.

Theorem 3.1. Let $d \geqslant N$. Let $\Theta^{(s)}, s \in \mathbb{N}$, be a sequence of natural lattices of degree $d-N$ in $\mathbb{R}^{N}$. We assume that $\Theta^{(s)}$ is the lattice given by the family of hyperplanes

$$
\mathbb{H}^{(s)}=\left\{\ell_{1}^{(s)}, \ldots, \ell_{d}^{(s)}\right\} \quad \text { with } \ell_{i}^{(s)}=\left\langle\mathbf{n}_{i}^{(s)}, \cdot\right\rangle-c_{i}^{(s)},\left\|\mathbf{n}_{i}^{(s)}\right\|=1, i=1, \ldots, d .
$$

Consider the following two conditions.

(C1) All the points of the lattice tend to 0 as $s \rightarrow \infty$, that is $\max \left\{\|\vartheta\|: \vartheta \in \Theta^{(s)}\right\} \rightarrow 0$ as $s \rightarrow \infty$.

(C2) The volumes

$$
\operatorname{vol}\left(\mathbf{n}_{i_{1}}^{(s)}, \ldots, \mathbf{n}_{i_{N}}^{(s)}\right), \quad 1 \leqslant i_{1}<i_{2}<\ldots<i_{N} \leqslant d,
$$

of the parallelotope spanned by the vectors $\mathbf{n}_{i_{1}}^{(s)}, \ldots, \mathbf{n}_{i_{N}}^{(s)}$ are bounded from below, away from 0 , uniformly in $s$.

If conditions (C1) and (C2) are satisfied then, for every function $f$ of class $C^{d-N+1}$ on a neighbourhood of the origin, we have

$$
\lim _{s \rightarrow \infty} \mathbf{L}\left[\Theta^{(s)} ; f\right]=\mathbf{T}_{0}^{d-N}(f) .
$$

Of course, (3.3) holds true in every normed vector space topology for $\mathcal{P}^{d-N}\left(\mathbb{R}^{N}\right)$.

\subsection{On condition (C2)}

The condition on the volume of the parallelotopes is equivalent to the following,

$$
\liminf _{s \rightarrow \infty} \min _{1 \leqslant i_{1}<\ldots<i_{N} \leqslant d}\left|\operatorname{det}\left(\mathbf{n}_{i_{1}}^{(s)}, \ldots, \mathbf{n}_{i_{N}}^{(s)}\right)\right|>0 .
$$

In $\mathbb{R}^{2}$ we have

$$
\operatorname{vol}\left(\mathbf{n}_{i}^{(s)}, \mathbf{n}_{j}^{(s)}\right)=\sin \left(\alpha_{i j}^{(s)}\right),
$$

where $\left.\alpha_{i j}^{(s)} \in\right] 0, \pi / 2\left[\right.$ is the line angle between the lines $\ell_{i}$ and $\ell_{j}$. Thus $\mathbb{H}$ satisfies condition $(\mathrm{C} 2)$ if and only if the angles between any two (distinct) lines in $\mathbb{H}^{(s)}$ remain uniformly bounded from below by a positive constant. An example of natural lattice of degree 2 in $\mathbb{R}^{2}$ and the various parameters involved in Theorem 3.1 are shown in Figure 1. 


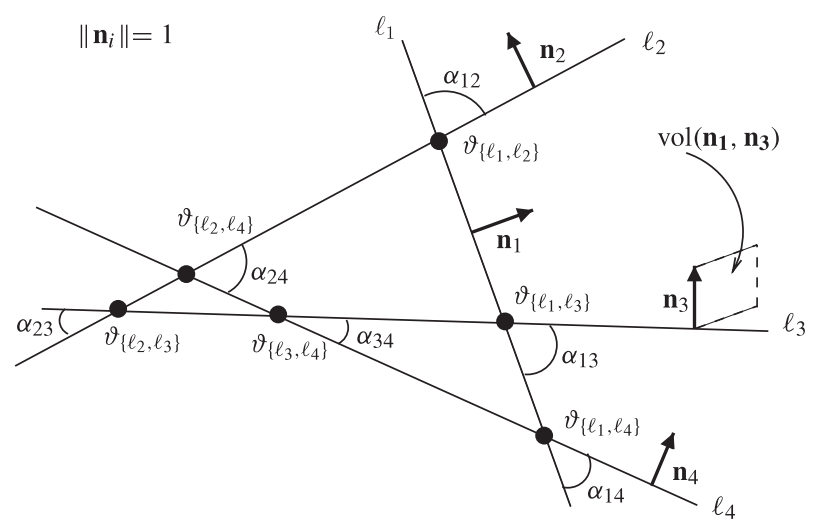

Figure 1. A bi-dimensional natural lattice.

Conditions (C1) and (C2) are independent. However, when we know that the second one holds true, the first one is easily checked as shown by the following lemma.

Lemma 3.2. If $(\mathrm{C} 2)$ is satisfied then $(\mathrm{C} 1)$ is equivalent to:

(C3) $\lim _{s \rightarrow \infty} \max _{i=1, \ldots, d}\left|c_{i}^{(s)}\right|=0$ where $c_{i}^{(s)}$ is defined in (3.1).

Proof. We show that (C1) implies (C3). Consider $H^{(s)} \in\left(\begin{array}{c}\mathbb{H}^{(s)} \\ N\end{array}\right)$ with $\ell_{i}^{(s)} \in H^{(s)}$. From $\left\langle\mathbf{n}_{i}^{(s)}, \vartheta_{H^{(s)}}\right\rangle-c_{i}^{(s)}=0$, we get

$$
\left|c_{i}^{(s)}\right| \leqslant\left\|\mathbf{n}_{i}^{(s)}\right\| \cdot\left\|\vartheta_{H^{(s)}}\right\|=\left\|\vartheta_{H^{(s)}}\right\| \rightarrow 0, \quad s \rightarrow \infty .
$$

To show the converse, we observe that if $H^{(s)}=\left\{\ell_{i_{1}}^{(s)}, \ldots, \ell_{i_{N}}^{(s)}\right\}$ then the coordinates $\left(x_{k}\right)$ of $\vartheta_{H^{(s)}}$ are solutions of the linear system

$$
\sum_{k=1}^{N} \mathbf{n}_{i_{j} k}^{(s)} x_{k}=c_{j}^{(s)}, \quad j=1, \ldots, N,
$$

and the claim follows from Cramer's formula in which, thanks to condition $(\mathrm{C} 2)$, the denominator remains away from 0 whereas the numerator tends to 0 .

\subsection{Affine transformations of natural lattices}

Let $\mathcal{L}(x)=\Lambda(x)+b$ be an affine transformation (isomorphism) of $\mathbb{R}^{N}$ with $\Lambda$ its linear part. If $\mathbb{H}$ is in general position so is $\mathcal{L}(\mathbb{H}):=\left\{\mathcal{L}\left(\ell_{i}\right): i=1, \ldots, d\right\}$ and $\mathcal{L}$ induces a one-to-one correspondence between $\left(\begin{array}{c}\mathbb{H} \\ N\end{array}\right)$ and $\left(\begin{array}{c}\mathcal{L}(\mathbb{H}) \\ N\end{array}\right)$. Moreover if $H \in\left(\begin{array}{c}\mathbb{H} \\ N\end{array}\right)$ then

$$
\vartheta_{\mathcal{L}(H)}=\mathcal{L}\left(\vartheta_{H}\right) \quad \text { and } \quad \Theta_{\mathcal{L}(\mathbb{H})}=\mathcal{L}\left(\Theta_{\mathbb{H}}\right) .
$$

In the following theorem we translate the conditions of Theorem 3.1 when the points of a natural lattice are sent to the origin by applying a sequence of affine transformations.

TheOREm 3.3. Let $\mathbb{H}=\left\{\ell_{1}, \ldots, \ell_{d}\right\}$ be a fixed collection of $d$ hyperplanes in general position in $\mathbb{R}^{N}, d \geqslant N$, with, as above, $\ell_{i}=\left\{x \in \mathbb{R}^{N}:\left\langle\mathbf{n}_{i}, x\right\rangle-c_{i}=0\right\},\left\|\mathbf{n}_{i}\right\|=1$. Let $\mathcal{L}_{s}=\Lambda_{s}+b_{s}$, $s \in \mathbb{N}$, be a sequence of affine transformations of $\mathbb{R}^{N}$. We set

$$
\mathbb{H}^{(s)}=\mathcal{L}_{s}(\mathbb{H}), \quad s \in \mathbb{N} .
$$


We consider the sequence of natural lattices $\Theta^{(s)}$ induced by $\mathbb{H}^{(s)}$. The following assertions are equivalent.

(i) The sequence $\Theta^{(s)}$ satisfies conditions $(\mathrm{C} 1)$ and $(\mathrm{C} 2)$.

(ii) There exists a positive constant $\Delta$ such that

$$
\left|\operatorname{det} \Lambda_{s}\right| \cdot \prod_{j=1}^{N}\left\|\Lambda_{s}^{-T}\left(\mathbf{n}_{i_{j}}\right)\right\| \leqslant \Delta, \quad 1 \leqslant i_{1}<\ldots<i_{N} \leqslant d, s \in \mathbb{N},
$$

and

$$
\max _{i=1, \ldots, d} \frac{1}{\left\|\Lambda_{s}^{-T}\left(\mathbf{n}_{i}\right)\right\|} \cdot\left|c_{i}+\left\langle\mathbf{n}_{i}, \Lambda_{s}^{-1}\left(b_{s}\right)\right\rangle\right| \rightarrow 0, \quad s \rightarrow \infty,
$$

where $\Lambda_{s}^{-T}$ denotes the transpose of the inverse of $\Lambda_{s}$.

Proof. It follows from the normalized equation of $\mathcal{L}_{s}\left(\ell_{i}\right)$ together with Lemma 3.2. Indeed, with $\ell_{i}(x)=\left\langle\mathbf{n}_{i}, x\right\rangle-c_{i}$, we have

$$
\mathcal{L}_{s}\left(\ell_{i}\right)=\left\{x \in \mathbb{R}^{N}:\left\langle\mathbf{n}_{i}, \mathcal{L}_{s}^{-1}(x)\right\rangle-c_{i}=0\right\} .
$$

Since for $x \in \mathcal{L}_{s}\left(\ell_{i}\right)$,

$$
\begin{aligned}
0 & =\left\langle\mathbf{n}_{i}, \Lambda_{s}^{-1}\left(x-b_{s}\right)\right\rangle-c_{i}=\left\langle\mathbf{n}_{i}, \Lambda_{s}^{-1}(x)\right\rangle-\left(c_{i}+\left\langle\mathbf{n}_{i}, \Lambda_{s}^{-1}\left(b_{s}\right)\right\rangle\right) \\
& =\left\langle\Lambda_{s}^{-T}\left(\mathbf{n}_{i}\right), x\right\rangle-\left(c_{i}+\left\langle\mathbf{n}_{i}, \Lambda_{s}^{-1}\left(b_{s}\right)\right\rangle\right)
\end{aligned}
$$

a normalized equation of $\mathcal{L}_{s}\left(\ell_{i}\right)$ is given by

$$
\left\langle\frac{\Lambda_{s}^{-T}\left(\mathbf{n}_{i}\right)}{\left\|\Lambda_{s}^{-T}\left(\mathbf{n}_{i}\right)\right\|}, x\right\rangle-\frac{1}{\left\|\Lambda_{s}^{-T}\left(\mathbf{n}_{i}\right)\right\|}\left\{c_{i}+\left\langle\mathbf{n}_{i}, \Lambda_{s}^{-1}\left(b_{s}\right)\right\rangle\right\} .
$$

\subsection{Examples}

In $\mathbb{R}^{2}$ any interpolation lattice of degree 1 is a natural lattice (based on the three distinct lines defined by the interpolation points). Moreover, any such lattice is the image under an affine isomorphism of the lattice $\Theta:=\{(0,0),(1,0),(0,1)\}$ constructed with the lines of equations $\ell_{1}\left(x_{1}, x_{2}\right)=x_{1}, \ell_{2}\left(x_{1}, x_{2}\right)=x_{2}$ and $\ell_{3}\left(x_{1}, x_{2}\right)=x_{1}+x_{2}-1$.

Consider the affine transformations $\mathcal{L}_{s}$ defined by

$$
\mathcal{L}_{s}(x)=\left(\begin{array}{cc}
t^{2} & 0 \\
0 & -t^{2} u
\end{array}\right)\left(\begin{array}{l}
x_{1} \\
x_{2}
\end{array}\right)+\left(\begin{array}{l}
t \\
t
\end{array}\right), \quad x=\left(\begin{array}{l}
x_{1} \\
x_{2}
\end{array}\right) \in \mathbb{R}^{2}, \quad t=1 / s, s \in \mathbb{N}^{\star},
$$

where $u$ is a function of $t$ such that $\lim _{t \rightarrow 0} u(t)=1$, and the lattice

$$
\Theta^{(s)}=\mathcal{L}_{s}(\Theta)=\left\{(t, t),\left(t^{2}+t, t\right),\left(t,-t^{2} u+t\right)\right\}, \quad t=1 / s .
$$

It is not difficult to see that $\Theta^{(s)}$ satisfies conditions $(\mathrm{C} 1)$ and $(\mathrm{C} 2)$. For $(\mathrm{C} 2)$ we use (3.5) and observe that one of the angles is equal to $\pi / 2$ while, thanks to the assumption on $u$, the other two tend to $\pi / 4$ as $t \rightarrow 0$. Hence, according to Theorem 3.1, the corresponding Lagrange interpolation polynomials at $\Theta^{(s)}$ of any twice continuously differentiable function $f$ on a neighbourhood of 0 converge to the Taylor polynomial of $f$. This example shows that the assumptions of Theorem 3.1, even in the simple case of Theorem 3.3, are weaker than those given in $[\mathbf{1}$, Proposition 2.1]. Indeed, the assumption

$$
\|(t, t)\|^{2} \cdot\left|\ell\left(\Theta^{(s)},(t, t), \cdot\right)\right| \rightarrow 0, \quad t \rightarrow 0,
$$

is required in that proposition whereas it clearly does not hold here since, as is easily checked,

$$
\ell\left(\Theta^{(s)},(t, t), x\right)=\frac{x_{2}-u x_{1}+\left(t^{2}+t\right) u-t}{t^{2} u} .
$$


We now give an example showing that convergence to the Taylor projector no longer holds, in general, when condition (C2) is not satisfied. We use a computation done in [1, Example 1.2]. We fix $\epsilon \geqslant 0$ and define

$$
\Theta_{\mathbb{H}(s)}=\left\{(0,0),\left(t, t^{2+\varepsilon}\right),(2 t, 0)\right\} \subset \mathbb{R}^{2}, \quad t=1 / s, s \in \mathbb{N}^{\star} .
$$

This lattice satisfies $(\mathrm{C} 1)$ but not $(\mathrm{C} 2)$ and it is readily checked that

$$
\mathbf{L}\left[\Theta_{\mathbb{H}(s)} ; X^{(2,0)}\right](x)=2 t x_{1}-\frac{x_{2}}{t^{\varepsilon}},
$$

which clearly does not converge to $\mathbf{T}_{0}^{1}\left(X^{(2,0)}\right)=0$ as $s=1 / t \rightarrow \infty$. The case $\epsilon=0$ shows that the Lagrange polynomials may converge to a limit different from the Taylor polynomial. Note that this does not mean that the sequence of operators $f \rightarrow \mathbf{L}\left[\Theta_{\mathbb{H}(s)} f\right]$ converge. For the possible limits of a sequence of Lagrange interpolation operators, we refer the reader to the works mentioned in the introduction.

\section{Further properties of natural lattices and proof of Theorem 3.1}

\section{1. de Boor's identity}

In the following, $\mathbb{H}$ always denotes a set of $d \geqslant N$ hyperplanes in general position in $\mathbb{R}^{N}$ and $\Theta=\Theta_{\mathbb{H}}$ the corresponding natural lattice. We will always assume that

$$
\mathbb{H}=\left\{\ell_{1}, \ldots, \ell_{d}\right\} .
$$

The elements of $\mathbb{H}$ are ordered according to the indexes. Every subset of $\mathbb{H}$ is endowed with the induced ordering.

If $K$ is a subset of $N-1$ elements in $\mathbb{H}$, that is $K \in\left(\begin{array}{c}\mathbb{H} \\ N-1\end{array}\right)$, then $\bigcap_{\ell \in K} \ell$ is a line in $\mathbb{R}^{N}$ which contains $d-N+1$ points of $\Theta$. Indeed, it passes through every $\vartheta_{H}$ such that $H \in\left(\begin{array}{c}\mathbb{H} \\ N\end{array}\right)$, $K \subset H$. The set of these $d-N+1$ points is denoted by $\Theta_{K}$,

$$
\Theta_{K}=\Theta \cap\left(\bigcap_{\ell \in K} \ell\right), \quad K \in\left(\begin{array}{c}
\mathbb{H} \\
N-1
\end{array}\right) \text {. }
$$

Assume that $K=\left\{\ell_{i_{1}}, \ldots, \ell_{i_{N-1}}\right\}$ with $i_{1}<i_{2}<\ldots<i_{N-1}$. Since the map

$$
v \in \mathbb{R}^{N} \mapsto \operatorname{det}\left(v, \mathbf{n}_{i_{1}}, \ldots, \mathbf{n}_{i_{N-1}}\right)
$$

is a linear form, there exists a vector, which we denote by $\mathbf{n}_{K}$, such that

$$
\operatorname{det}\left(v, \mathbf{n}_{i_{1}}, \ldots, \mathbf{n}_{i_{N-1}}\right)=\left\langle v, \mathbf{n}_{K}\right\rangle, \quad v \in \mathbb{R}^{N} .
$$

As defined, the value of $\mathbf{n}_{K}$ depends on the ordering of the hyperplanes of $K$. A different ordering may change $\mathbf{n}_{K}$ into $-\mathbf{n}_{K}$. It is to avoid further discussion of this detail that we assumed we start with a particular ordering of $\mathbb{H}$ and agreed that every subset of $\mathbb{H}$ is endowed with the induced ordering.

Lemma 4.1. The direction of the line $\bigcap_{\ell \in K} \ell$ is given by the (nonzero) vector $\mathbf{n}_{K}$. We have $\left\|\mathbf{n}_{K}\right\| \leqslant 1$.

Proof. Let $K=\left\{\ell_{i_{1}}, \ldots, \ell_{i_{N-1}}\right\}$. The first claim is a consequence of the equations

$$
\left\langle\mathbf{n}_{i_{j}}, \mathbf{n}_{K}\right\rangle=0, \quad j=1, \ldots, N-1,
$$

which follows readily from (4.4). Next, by Hadamard's inequality, the norm of the linear form (4.3) is smaller than the product of the lengths $\left\|\mathbf{n}_{i_{j}}\right\|$, which is smaller than one. Hence, in view of (4.4), so is the norm of $\mathbf{n}_{K}$. 
The vectors $\mathbf{n}_{K}$ play a fundamental role in our proof of Theorem 3.1.

Note in particular that if $H \in\left(\begin{array}{c}\mathbb{H} \\ N\end{array}\right)$ and $\ell \in H$ then we may speak of $\mathbf{n}_{H \backslash \ell}$. From now on, we use $H \backslash \ell_{i}$ for $H \backslash\left\{\ell_{i}\right\}$.

LEMma 4.2 (de Boor's identity). If $H \in\left(\begin{array}{l}\mathbb{H} \\ N\end{array}\right)$ then we have

$$
x=\vartheta_{H}+\sum_{\ell \in H} \frac{\ell(x)}{\tilde{\ell}\left(\mathbf{n}_{H \backslash \ell}\right)} \mathbf{n}_{H \backslash \ell}, \quad x \in \mathbb{R}^{N},
$$

where $\tilde{\ell}$ denotes the linear part of $\ell$ (thus $\tilde{\ell}(x)=\langle\mathbf{n}, x\rangle$ if $\ell(x)=\langle\mathbf{n}, x\rangle-c)$. In particular, for every $H$, the vectors $\mathbf{n}_{H \backslash \ell}, \ell \in H$, form a basis of $\mathbb{R}^{N}$.

Proof. See [4, p. 37].

4.2. de Boor's remainder formula

We now recall the definition of multivariate divided differences. Let $\Omega$ be an open convex set in $\mathbb{R}^{N}$; to every set $A=\left\{a_{0}, \ldots, a_{s}\right\} \subset \Omega$ (the points are not necessarily distinct) and $f \in C^{s}(\Omega)$, we associate a $s$-linear form on $\left(\mathbb{R}^{N}\right)^{s}$ defined by

$$
\begin{aligned}
& \left(\mathbb{R}^{N}\right)^{s} \ni\left(v_{1}, \ldots, v_{s}\right) \longmapsto \\
& \quad\left[a_{0}, \ldots, a_{s} \mid v_{1}, \ldots, v_{s}\right] f:=\int_{[A]} D_{v_{1}} \ldots D_{v_{s}} f=\int_{[A]} f^{(s)}(\cdot)\left(v_{1}, \ldots, v_{s}\right),
\end{aligned}
$$

where $f^{(s)}$ denotes the $s$ th total derivative of $f$,

$$
\int_{[A]} g=\int_{\Delta_{s}} g\left(a_{0}+\sum_{i=1}^{s} \xi_{i}\left(a_{i}-a_{0}\right)\right) d \xi_{1} \ldots d \xi_{s}
$$

and $\Delta_{s}$ is the standard simplex $\left\{\xi=\left(\xi_{1}, \ldots, \xi_{s}\right): \xi_{i} \geqslant 0, \sum_{i=1}^{s} \xi_{i} \leqslant 1\right\}$. This symmetric $s$-linear form is called the multivariate divided difference of $f$ at $A$. Note that, when $f \in C^{s}(\Omega)$ is fixed, the function

$$
\Omega^{s+1} \times\left(\mathbb{R}^{N}\right)^{s} \ni\left(a_{0}, \ldots, a_{s}, v_{1}, \ldots, v_{s}\right) \longmapsto\left[a_{0}, \ldots, a_{s} \mid v_{1}, \ldots, v_{s}\right] f
$$

is continuous (as a function of its two groups of variables).

We now state a beautiful error formula due to Carl de Boor.

THEOREM 4.3 (de Boor's remainder formula). Let $\mathbb{H}=\left\{\ell_{1}, \ldots, \ell_{d}\right\}$ be a collection of $d \geqslant N$ hyperplanes in general position in $\mathbb{R}^{N}$ and $\Theta=\Theta_{\mathbb{H}}$ the corresponding natural lattice. For $K \in\left(\begin{array}{c}\mathbb{H} \\ N-1\end{array}\right)$, we define the polynomial $P_{K}$ of degree $d-N+1$ by the relation

$$
P_{K}(x)=\prod_{\ell \in \mathbb{H} \backslash K} \frac{\ell(x)}{\tilde{\ell}\left(\mathbf{n}_{K}\right)},
$$

where, as above, $\tilde{\ell}$ is used for the linear part of $\ell$.

The error between a function $f$ of class $C^{d-N+1}$ on a convex neighbourhood $\Omega$ of $\Theta$ and the Lagrange interpolation polynomial of $f$ at $\Theta$ is given by the following formula:

$$
f(x)=\mathbf{L}[\Theta ; f](x)+\sum_{K \in\left(\begin{array}{c}
H \\
N-1
\end{array}\right)} P_{K}(x) \cdot[\Theta_{K}, x \mid \underbrace{\mathbf{n}_{K}, \ldots, \mathbf{n}_{K}}_{d-N+1}] f, \quad x \in \Omega .
$$

Recall that for $K \in\left(\begin{array}{c}\mathbb{H} \\ N-1\end{array}\right), \Theta_{K}$ is the subset formed by the $d-N+1$ points of $\Theta$ lying on the line $\bigcap_{\ell \in K} \ell$, see $(4.2)$.

Proof. See [4, Theorem 3.1]. 


\subsection{Some algebraic identities}

We now prove two auxiliary lemmas. The first one (Lemma 4.4) shows that the points $\mathbf{n}_{K}$, $K \in\left(\begin{array}{c}\mathbb{H} \\ N-1\end{array}\right)$, themselves form a certain interpolation lattice. The second one (Lemma 4.6) is a somewhat mysterious representation formula for symmetric multi-linear forms.

Lemma 4.4. Let $\mathbb{H}=\left\{\ell_{1}, \ldots, \ell_{d}\right\}$ be a collection of $d$ hyperplanes in general position in $\mathbb{R}^{N}$ with $d \geqslant N$. The set

$$
\mathcal{V}:=\left\{\mathbf{n}_{K}: K \in\left(\begin{array}{c}
\mathbb{H} \\
N-1
\end{array}\right)\right\}
$$

is an interpolation lattice for the space $\mathcal{H}^{d-N+1}\left(\mathbb{R}^{N}\right)$ of homogeneous polynomials of degree $d-N+1$.

Proof. It suffices to prove the following two assertions.

(i) The cardinality of $\mathcal{V}$ is equal to the dimension of $\mathcal{H}^{d-N+1}\left(\mathbb{R}^{N}\right)$ which is $\left(\begin{array}{c}d \\ d-N+1\end{array}\right)=\left(\begin{array}{c}d \\ N-1\end{array}\right)$.

(ii) For every $\mathbf{n}_{K}$ in $\mathcal{V}$ there exists $H_{K} \in \mathcal{H}^{d-N+1}\left(\mathbb{R}^{N}\right)$ such that $H_{K}\left(\mathbf{n}_{K}\right)=1$ but $H_{K}$ vanishes on $\mathcal{V} \backslash\left\{\mathbf{n}_{K}\right\}$.

To verify the first point, we just need to check that if $K, K^{\prime} \in\left(\begin{array}{c}\mathbb{H} \\ N-1\end{array}\right)$ and $K \neq K^{\prime}$ then $\mathbf{n}_{K} \neq \mathbf{n}_{K^{\prime}}$. But, if $K \neq K^{\prime}$ there exists $\ell \in K \backslash K^{\prime}$ with $\ell(x)=\langle\mathbf{n}, x\rangle-c$. Assume that $K^{\prime}=\left\{\ell_{i_{1}}, \ldots, \ell_{i_{N-1}}\right\}$. Since $\ell \cup K^{\prime}$ is a set of $N$ hyperplanes in general position, we have $\operatorname{det}\left(\mathbf{n}, \mathbf{n}_{i_{1}}, \ldots, \mathbf{n}_{i_{N-1}}\right) \neq 0$ hence, in view of (4.4), $\left\langle\mathbf{n}, \mathbf{n}_{K^{\prime}}\right\rangle \neq 0$. On the other hand, since $\ell \in K$, $\left\langle\mathbf{n}, \mathbf{n}_{K}\right\rangle=0$. Hence $\mathbf{n}_{K} \neq \mathbf{n}_{K^{\prime}}$.

As for the second point, for $K \in\left(\begin{array}{c}\mathbb{H} \\ N-1\end{array}\right)$, we set

$$
H_{K}:=\tilde{P}_{K}(x)=\prod_{\ell \in \mathbb{H} \backslash K} \frac{\tilde{\ell}(x)}{\tilde{\ell}\left(\mathbf{n}_{K}\right)} .
$$

This clearly defines a homogeneous polynomial of degree $d-N+1$ in $\mathbb{R}^{N}$ satisfying $H_{K}\left(\mathbf{n}_{K}\right)=1$. Moreover, if $K^{\prime} \in\left(\begin{array}{c}\mathbb{H} \\ N-1\end{array}\right), K^{\prime} \neq K$, then we can find $\ell$ in $(\mathbb{H} \backslash K) \cap K^{\prime}$. Since $\ell \notin K$, the factor $\tilde{\ell}\left(\mathbf{n}_{K^{\prime}}\right)$ appears in $H_{K}\left(\mathbf{n}_{K^{\prime}}\right)$. However, since $\ell \in K^{\prime}, \tilde{\ell}\left(\mathbf{n}_{K^{\prime}}\right)=\left\langle\mathbf{n}, \mathbf{n}_{K^{\prime}}\right\rangle=0$. Hence $H_{K}\left(\mathbf{n}_{K^{\prime}}\right)=0$.

Applying the interpolation formula corresponding to the above interpolation lattice to the homogeneous polynomial $v \rightarrow \phi\left(v^{d-N+1}\right)$ where $\phi$ is a symmetric multi-linear form we obtain the following identity. We use the polynomials $H_{K}=\tilde{P}_{K}$ in (4.10).

Corollary 4.5. With the assumptions of the lemma, for every symmetric $(d-N+1)$ linear form $\phi$ on $\mathbb{R}^{N}$, we have

$$
\phi\left(v^{d-N+1}\right)=\sum_{K \in\left(\begin{array}{c}
\mathrm{H} \\
N-1
\end{array}\right)} \tilde{P}_{K}(v) \cdot \phi\left(\mathbf{n}_{K}^{d-N+1}\right), \quad v \in \mathbb{R}^{N},
$$

where we use $u^{d-N+1}:=(u, \ldots, u)(d-N+1$ times $)$.

Lemma 4.6. Let $\mathbb{H}=\left\{\ell_{1}, \ldots, \ell_{d}\right\}$ be a collection of hyperplanes in $\mathbb{R}^{N}$ in general position with $d \geqslant N$. We set

$$
\mathbb{H}_{i}=\left\{\ell_{1}, \ldots, \ell_{i}\right\}, \quad 1 \leqslant i \leqslant d,
$$

and

$$
P_{K}^{[i-1]}(x)=\prod_{\ell \in \mathbb{H}_{i-1} \backslash K} \frac{\ell(x)}{\tilde{\ell}\left(\mathbf{n}_{K}\right)}, \quad K \in\left(\begin{array}{c}
\mathbb{H}_{i-1} \\
N-1
\end{array}\right), \quad N \leqslant i \leqslant d+1 .
$$


Then for every symmetric $(d-N+1)$-linear form $\phi$ on $\mathbb{R}^{N}$, we have

$$
\phi\left(x^{d-N+1}\right)=\sum_{i=N}^{d+1} \sum_{K \in\left(\begin{array}{c}
\mathbb{H}_{i-1} \\
N-1
\end{array}\right)} P_{K}^{[i-1]}(x) \cdot \phi\left(x^{d-i}, \vartheta_{K \cup \ell_{i}}, \mathbf{n}_{K}^{i-N}\right), \quad x \in \mathbb{R}^{N} .
$$

The notation in the above formula must be understood as follows:

(1) when $d-i$ (respectively $i-N)$ is not positive then $x$ (respectively $\mathbf{n}_{K}$ ) does not appear in $\phi\left(x^{d-i}, \vartheta_{K \cup \ell_{i}}, \mathbf{n}_{K}^{i-N}\right)$; and

(2) when $i=d+1$ then $x$ and $\vartheta_{K \cup \ell_{i}}$ do not appear;

(3) likewise, if the product in the definition of $P_{K}^{[i-1]}$ is empty then its value is taken to be 1. (This happens when $i=N$.)

To deal with the case $N \geqslant 3$ we need the following simple observation.

Lemma 4.7. Let $\mathbb{H}=\left\{\ell_{1}, \ldots, \ell_{d+1}\right\}$ be a collection of hyperplanes in $\mathbb{R}^{N}$ in general position with $d \geqslant N \geqslant 3$. As above, we write $\mathbb{H}_{d}=\left\{\ell_{1}, \ldots, \ell_{d}\right\}$. Let $K^{\prime} \in\left(\begin{array}{c}\mathbb{H}_{d} \\ N-2\end{array}\right)$. If $K \in\left(\begin{array}{c}\mathbb{H}_{d} \\ N-1\end{array}\right)$ and $K^{\prime} \nsubseteq K$ then

$$
\tilde{P}_{K}^{[d]}\left(\mathbf{n}_{K^{\prime} \cup \ell_{d+1}}\right)=0 \quad \text { where } \tilde{P}_{K}^{[d]}=\prod_{\ell \in \mathbb{H}_{d} \backslash K} \frac{\tilde{\ell}(\cdot)}{\tilde{\ell}\left(\mathbf{n}_{K}\right)} .
$$

Proof. Take $\ell_{i} \in K^{\prime} \cap\left(\mathbb{H}_{d} \backslash K\right)$. The fact that $\ell_{i} \in K^{\prime}$ gives

$$
0=\left\langle\mathbf{n}_{i}, \mathbf{n}_{K^{\prime} \cup \ell_{d+1}}\right\rangle=\tilde{\ell}_{i}\left(\mathbf{n}_{K^{\prime} \cup \ell_{d+1}}\right)
$$

and since $\ell_{i} \in \mathbb{H}_{d} \backslash K$ it follows that $\tilde{\ell}_{i}\left(\mathbf{n}_{K^{\prime} \cup \ell_{d+1}}\right)$ is a factor of $\tilde{P}_{K}^{[d]}\left(\mathbf{n}_{K^{\prime} \cup \ell_{d+1}}\right)$.

Proof of Lemma 4.6. We prove identity (4.14) by induction on $d \geqslant N$.

(A) We start with the case $d=N$. In that case (4.14) reduces to

$$
\begin{aligned}
\phi(x) & =\sum_{K \in\left(\begin{array}{c}
\mathbb{H}_{N}-1 \\
N-1
\end{array}\right)} P_{K}^{[N-1]}(x) \phi\left(\vartheta_{K \cup \ell_{N}}\right)+\sum_{K \in\left(\begin{array}{c}
\mathbb{H}_{N} \\
N-1
\end{array}\right)} P_{K}^{[N]}(x) \phi\left(\mathbf{n}_{K}\right) \\
& =\phi\left(\vartheta_{\mathbb{H}_{N}}\right)+\sum_{i=1}^{N} \frac{\ell_{i}(x)}{\tilde{\ell}_{i}\left(\mathbf{n}_{\mathbb{H}_{N} \backslash \ell_{i}}\right)} \phi\left(\mathbf{n}_{\mathbb{H}_{N} \backslash \ell_{i}}\right) .
\end{aligned}
$$

Since $\phi$ is a linear form, the claim follows from de Boor's identity (4.5).

(B) We assume that (4.14) holds true for $d$ and prove it for $d+1$. Take $\phi$ a symmetric $(d+2-N)$-linear form. Fix $y \in \mathbb{R}^{N}$ and define $\phi_{y}$ on $\left(\mathbb{R}^{N}\right)^{d+1-N}$ by

$$
\phi_{y}\left(v_{1}, \ldots, v_{d+1-N}\right)=\phi\left(v_{1}, \ldots, v_{d+1-N}, y\right) .
$$

Thus $\phi_{y}$ is a symmetric $(d+1-N)$-linear form to which we may apply the induction hypothesis to get

$$
\phi_{y}\left(x^{d-N+1}\right)=\sum_{i=N}^{d+1} \sum_{K \in\left(\begin{array}{c}
\mathbb{H}_{i}-1 \\
N-1
\end{array}\right)} P_{K}^{[i-1]}(x) \phi_{y}\left(x^{d-i}, \vartheta_{K \cup \ell_{i}}, \mathbf{n}_{K}^{i-N}\right) .
$$

Putting $y=x$ in the above expression, we obtain

$$
\phi\left(x^{d-N+2}\right)=\sum_{i=N}^{d+1} \sum_{K \in\left(\begin{array}{c}
\mathbb{H}_{i}-1 \\
N-1
\end{array}\right)} P_{K}^{[i-1]}(x) \phi_{x}\left(x^{d-i}, \vartheta_{K \cup \ell_{i}}, \mathbf{n}_{K}^{i-N}\right) .
$$


On the other hand, we need to prove

$$
\phi\left(x^{d-N+2}\right)=\sum_{i=N}^{d+2} \sum_{K \in\left(\begin{array}{l}
\mathbb{H}_{i}-1 \\
N-1
\end{array}\right)} P_{K}^{[i-1]}(x) \phi\left(x^{d+1-i}, \vartheta_{K \cup \ell_{i}}, \mathbf{n}_{K}^{i-N}\right) .
$$

Expressions (4.18) and (4.19) differ only for $i=d+2$ and $i=d+1$ (observe that $x$ appears for $i=d+1$ in (4.18) but not in (4.19). Thus, to establish (4.19), it suffices to prove that the term corresponding to $d+1$ in (4.18) equals the sum of the terms corresponding to $d+1$ and $d+2$ in (4.19), that is

$$
\begin{aligned}
\sum_{K \in\left(\begin{array}{l}
\mathbb{H}_{d} \\
N-1
\end{array}\right)} P_{K}^{[d]}(x) \phi\left(\mathbf{n}_{K}^{d+1-N}, x\right)= & \sum_{K \in\left(\begin{array}{l}
\mathbb{H}_{d} \\
N-1
\end{array}\right)} P_{K}^{[d]}(x) \phi\left(\vartheta_{K \cup \ell_{d+1}}, \mathbf{n}_{K}^{d+1-N}\right) \\
& +\sum_{K \in\left(\begin{array}{l}
\mathbb{H}_{d}+1 \\
N-1
\end{array}\right)} P_{K}^{[d+1]}(x) \phi\left(\mathbf{n}_{K}^{d+2-N}\right) .
\end{aligned}
$$

For $K \in\left(\begin{array}{c}\mathbb{H}_{d} \\ N-1\end{array}\right)$ and $x \in \mathbb{R}^{N}$, using de Boor's identity (4.5) with $H=K \cup \ell_{d+1}$, we may write

$$
\begin{aligned}
x & =\vartheta_{K \cup \ell_{d+1}}+\sum_{\ell \in K \cup \ell_{d+1}} \frac{\ell(x)}{\tilde{\ell}\left(\mathbf{n}_{\left(K \cup \ell_{d+1}\right) \backslash \ell}\right)} \mathbf{n}_{\left(K \cup \ell_{d+1}\right) \backslash \ell} \\
& =\vartheta_{K \cup \ell_{d+1}}+\frac{\ell_{d+1}(x)}{\tilde{\ell}_{d+1}\left(\mathbf{n}_{K}\right)} \mathbf{n}_{K}+\sum_{\ell \in K} \frac{\ell(x)}{\tilde{\ell}\left(\mathbf{n}_{(K \backslash \ell) \cup \ell_{d+1}}\right)} \mathbf{n}_{(K \backslash \ell) \cup \ell_{d+1}} .
\end{aligned}
$$

Substituting $x$ with the above expression in the $\phi$-term of the left-hand side of (4.20), we arrive to

$$
\begin{aligned}
\sum_{K \in\left(\begin{array}{c}
\mathbb{H}_{d} \\
N-1
\end{array}\right)} P_{K}^{[d]}(x) \cdot \phi\left(\mathbf{n}_{K}^{d+1-N}, x\right)= & \sum_{K \in\left(\begin{array}{c}
\mathbb{H}_{d} \\
N-1
\end{array}\right)} P_{K}^{[d]}(x) \cdot \phi\left(\vartheta_{K \cup \ell_{d+1}}, \mathbf{n}_{K}^{d+1-N}\right) \\
& +\sum_{K \in\left(\begin{array}{c}
\mathbb{H}_{d} \\
N-1
\end{array}\right)} P_{K}^{[d]}(x) \frac{\ell_{d+1}(x)}{\tilde{\ell}_{d+1}\left(\mathbf{n}_{K}\right)} \cdot \phi\left(\mathbf{n}_{K}^{d+2-N}\right) \\
& +\sum_{\substack{K \in\left(\begin{array}{c}
\mathbb{H}_{d} \\
N-1
\end{array}\right) \\
\sum_{\ell \in K}}} P_{K}^{[d]}(x) \frac{\ell(x)}{\tilde{\ell}\left(\mathbf{n}_{(K \backslash \ell) \cup \ell_{d+1}}\right)} \\
& \cdot \phi\left(\mathbf{n}_{K}^{d+1-N}, \mathbf{n}_{(K \backslash \ell) \cup \ell_{d+1}}\right) .
\end{aligned}
$$

Now, for $K \in\left(\begin{array}{c}\mathbb{H}_{d} \\ N-1\end{array}\right)$, we have

$$
P_{K}^{[d]}(x) \frac{\ell_{d+1}(x)}{\tilde{\ell}_{d+1}\left(\mathbf{n}_{K}\right)}=P_{K}^{[d+1]}(x) .
$$

Hence, we may regard the second term on the right-hand side of (4.21) as a sum over $\left(\begin{array}{l}\mathbb{H}_{d+1} \\ N-1\end{array}\right)$ to obtain

$$
\sum_{K \in\left(\begin{array}{c}
\mathbb{H}_{d+1} \\
N-1
\end{array}\right), \ell_{d+1} \notin K} P_{K}^{[d+1]}(x) \cdot \phi\left(\mathbf{n}_{K}^{d+2-N}\right) .
$$

Thus, since $K \in\left(\begin{array}{c}\mathbb{H}_{d+1} \\ N-1\end{array}\right), \ell_{d+1} \in K$ means $K=K^{\prime} \cup\left\{\ell_{d+1}\right\}$ with $K^{\prime} \in\left(\begin{array}{c}\mathbb{H}_{d} \\ N-2\end{array}\right)$; to prove (4.20), it remains to establish

$$
\begin{aligned}
& \sum_{K \in\left(\begin{array}{c}
\mathbb{H} d \\
N-1
\end{array}\right)} \sum_{\ell \in K} P_{K}^{[d]}(x) \frac{\ell(x)}{\tilde{\ell}\left(\mathbf{n}_{(K \backslash \ell) \cup \ell_{d+1}}\right)} \cdot \phi\left(\mathbf{n}_{K}^{d+1-N}, \mathbf{n}_{(K \backslash \ell) \cup \ell_{d+1}}\right) \\
& =\sum_{K^{\prime} \in\left(\begin{array}{c}
\mathbb{H}_{d} \\
N-2
\end{array}\right)} P_{K^{\prime} \cup \ell_{d+1}}^{[d+1]}(x) \cdot \phi\left(\mathbf{n}_{K^{\prime} \cup \ell_{d+1}^{d+2-N}}\right) .
\end{aligned}
$$


Observe that in the case $N=2$, since $K^{\prime}=\emptyset$, the sum on the right-hand side reduces to a single element.

We first concentrate on the term

$$
P_{K}^{[d]}(x) \frac{\ell(x)}{\tilde{\ell}\left(\mathbf{n}_{(K \backslash \ell) \cup \ell_{d+1}}\right)}=\left\{\prod_{h \in \mathbb{H}_{d} \backslash K} \frac{h(x)}{\tilde{h}\left(\mathbf{n}_{K}\right)}\right\} \cdot \frac{\ell(x)}{\tilde{\ell}\left(\mathbf{n}_{(K \backslash \ell) \cup \ell_{d+1}}\right)}
$$

on the left-hand side of (4.23). Since $\ell \in K$ we have

$$
\begin{aligned}
P_{K}^{[d]}(x) \frac{\ell(x)}{\tilde{\ell}\left(\mathbf{n}_{\left.(K \backslash \ell) \cup \ell_{d+1}\right)}\right)} & =\prod_{\substack{h \in \mathbb{H}_{d} \backslash(K \backslash \ell) \\
\tilde{h}\left(\mathbf{n}_{(K \backslash \ell) \cup \ell_{d+1}}\right)}} \frac{h(x)}{\prod_{h \in \mathbb{H}_{d} \backslash K}} \frac{\tilde{h}\left(\mathbf{n}_{(K \backslash \ell) \cup \ell_{d+1}}\right)}{\tilde{h}\left(\mathbf{n}_{K}\right)} \\
& =P_{(K \backslash \ell) \cup \ell_{d+1}}^{[d+1]}(x) \cdot \tilde{P}_{K}^{[d]}\left(\mathbf{n}_{(K \backslash \ell) \cup \ell_{d+1}}\right) .
\end{aligned}
$$

We now use this expression in the left-hand side of (4.23). Observing again that when $K$ runs over $\left(\begin{array}{c}\mathbb{H}_{d} \\ N-1\end{array}\right)$ and $\ell$ runs over $K$ then $K \backslash \ell$ runs over $\left(\begin{array}{c}\mathbb{H}_{d} \\ N-2\end{array}\right)$ we arrive at

$$
\begin{aligned}
& \sum_{K \in\left(\begin{array}{c}
\mathbb{H}_{N} \\
N-1
\end{array}\right)} \sum_{\ell \in K} P_{K}^{[d]}(x) \frac{\ell(x)}{\tilde{\ell}\left(\mathbf{n}_{(K \backslash \ell) \cup \ell_{d+1}}\right)} \cdot \phi\left(\mathbf{n}_{K}^{d-N+1}, \mathbf{n}_{(K \backslash \ell) \cup \ell_{d+1}}\right) \\
& =\sum_{K \in\left(\begin{array}{c}
\mathbb{H}_{d} \\
N-1
\end{array}\right)} \sum_{\ell \in K} P_{(K \backslash \ell) \cup \ell_{d+1}}^{[d+1]}(x) \tilde{P}_{K}^{[d]}\left(\mathbf{n}_{(K \backslash \ell) \cup \ell_{d+1}}\right) \cdot \phi\left(\mathbf{n}_{K}^{d-N+1}, \mathbf{n}_{(K \backslash \ell) \cup \ell_{d+1}}\right) \\
& =\sum_{K^{\prime} \in\left(\begin{array}{c}
\mathbb{H}_{d} \\
N-2
\end{array}\right)} P_{K^{\prime} \cup \ell_{d+1}}^{[d+1]}(x) \sum_{K^{\prime} \subset K \in\left(\begin{array}{c}
\mathbb{H}_{d} d \\
N-1
\end{array}\right)} \tilde{P}_{K}^{[d]}\left(\mathbf{n}_{K^{\prime} \cup \ell_{d+1}}\right) \cdot \phi\left(\mathbf{n}_{K}^{d-N+1}, \mathbf{n}_{K^{\prime} \cup \ell_{d+1}}\right) .
\end{aligned}
$$

Now, for a fixed $K^{\prime} \in\left(\begin{array}{c}\mathbb{H}_{d} \\ N-2\end{array}\right)$, using Lemma 4.7 for the first equality in the case $N \geqslant 3$ (we add null terms) and Corollary 4.5 for the second one, we get

$$
\begin{aligned}
& \sum_{K^{\prime} \subset K \in\left(\begin{array}{c}
\mathbb{H}_{N} d \\
N-1
\end{array}\right)} \tilde{P}_{K}^{[d]}\left(\mathbf{n}_{K^{\prime} \cup \ell_{d+1}}\right) \phi\left(\mathbf{n}_{K}^{d-N+1}, \mathbf{n}_{K^{\prime} \cup \ell_{d+1}}\right) \\
= & \sum_{K \in\left(\begin{array}{c}
\mathbb{H}_{d} \\
N-1
\end{array}\right)} \tilde{P}_{K}^{[d]}\left(\mathbf{n}_{K^{\prime} \cup \ell_{d+1}}\right) \phi\left(\mathbf{n}_{K}^{d-N+1}, \mathbf{n}_{K^{\prime} \cup \ell_{d+1}}\right)=\phi\left(\mathbf{n}_{K^{\prime} \cup \ell_{d+1}^{d-2}}^{d-N+2}\right) .
\end{aligned}
$$

Using (4.27) in the last term of (4.26), we finally arrive at

$$
\begin{aligned}
& \sum_{K \in\left(\begin{array}{c}
\mathbb{H}_{d} \\
N-1
\end{array}\right)} \sum_{\ell \in K} P_{K}^{[d]}(x) \frac{\ell(x)}{\tilde{\ell}\left(\mathbf{n}_{(K \backslash \ell) \cup \ell_{d+1}}\right)} \phi\left(\mathbf{n}_{K}^{d-N+1}, \mathbf{n}_{(K \backslash \ell) \cup \ell_{d+1}}\right) \\
& \quad=\sum_{K^{\prime} \in\left(\begin{array}{l}
\mathbb{H}_{d} \\
N-2
\end{array}\right)} P_{K^{\prime} \cup \ell_{d+1}}^{[d+1]}(x) \cdot \phi\left(\mathbf{n}_{K^{\prime} \cup \ell_{d+1}}^{d-N+2}\right),
\end{aligned}
$$

which is (4.23). This completes the proof of the lemma.

Corollary 4.8. Let $\mathbb{H}=\left\{\ell_{1}, \ldots, \ell_{d}\right\}$ be a collection of $d \geqslant N$ hyperplanes in general position in $\mathbb{R}^{N}$. For every function $f$ of class $C^{d-N+1}$ on a convex neighbourhood $\Omega$ of the origin in $\mathbb{R}^{N}$ we have

$$
\begin{aligned}
& f(x)-\mathbf{T}_{0}^{d-N}(f)(x) \\
& \quad=\sum_{i=N}^{d+1} \sum_{K \in\left(\begin{array}{c}
\mathbb{H}_{i-1}-1 \\
N-1
\end{array}\right)} P_{K}^{[i-1]}(x) \cdot \int_{[\underbrace{0, \ldots, 0, x]}_{d-N+1}} f^{(d-N+1)}(\cdot)\left(x^{d-i}, \vartheta_{K \cup \ell_{i}}, \mathbf{n}_{K}^{i-N}\right), \quad x \in \Omega .
\end{aligned}
$$


Proof. The remainder formula for Taylor polynomials (as a special case of Kergin interpolation, see for example [7, Theorem 3]) gives us,

$$
f(x)-\mathbf{T}_{0}^{d-N}(f)(x)=[\underbrace{0, \ldots, 0}_{d-N+1}, x \mid \underbrace{x, \ldots, x}_{d-N+1}] f=\int_{[0, \ldots, 0, x]} f^{(d-N+1)}(\cdot)(x, \ldots, x) .
$$

The corollary then follows directly from Lemma 4.6 since, for every $a \in \Omega, f^{(d-N+1)}(a)$ is a symmetric $(d-N+1)$-linear form on $\mathbb{R}^{N}$.

\subsection{Proof of Theorem 3.1}

Let $\Omega$ be a neighbourhood of the origin on which $f$ is of class $C^{d+1}$. We may assume that:

(i) $\Omega$ contains $B(0, R)$, the closed euclidean ball of centre the origin and radius $R$ and, in view of condition $(\mathrm{C} 1)$;

(ii) all the points of $\Theta^{(s)}=\Theta_{\mathbb{H}^{(s)}}$ lie in $B(0, R), s \in \mathbb{N}$.

We set

$$
M=\max _{a \in B(0, R)}\left\|f^{(d-N+1)}(a)\right\|<\infty,
$$

where $\|\cdot\|$ here denotes the usual norm of a multi-linear form. We use condition (C2) in the form given by (3.4) taking (4.4) into account as follows.

(iii) There exists $\delta>0$ such that

$$
\left|\left\langle\mathbf{n}_{i}, \mathbf{n}_{K}\right\rangle\right| \geqslant \delta, \quad K \in\left(\begin{array}{c}
\mathbb{H}^{(s)} \\
N-1
\end{array}\right), \quad \ell_{i} \notin K, s \in \mathbb{N} .
$$

(A) We first derive an estimate on the polynomials $P_{K}=P_{K}^{[d]}$ defined in (4.7). We claim that

$$
\left|P_{K}^{[d]}(x)\right| \leqslant\left(\frac{2 R}{\delta}\right)^{d-N+1}, \quad x \in B(0, R), \quad K \in\left(\begin{array}{c}
\mathbb{H}^{(s)} \\
N-1
\end{array}\right), \quad s \in \mathbb{N} .
$$

Indeed, if $K \in\left(\begin{array}{l}\mathbb{H}_{N-1}^{(s)} \\ N-1\end{array}\right)$ and $\ell_{i} \in \mathbb{H}^{(s)} \backslash K$, since $\vartheta_{K \cup \ell_{i}} \in \ell_{i}$, we have

$$
\left|c_{i}\right|=\left|\left\langle\mathbf{n}_{i}, \vartheta_{K \cup \ell_{i}}\right\rangle\right| \leqslant\left\|\vartheta_{K \cup \ell_{i}}\right\| \leqslant R .
$$

Next, using (4.31) and $\left\|\mathbf{n}_{i}\right\|=1$, we have

$$
\left|\frac{\ell_{i}(x)}{\tilde{\ell}_{i}\left(\mathbf{n}_{K}\right)}\right| \leqslant \frac{\left|\left\langle\mathbf{n}_{i}, x\right\rangle\right|+\left|c_{i}\right|}{\left|\left\langle\mathbf{n}_{i}, \mathbf{n}_{K}\right\rangle\right|} \leqslant \frac{2 R}{\delta}, \quad \ell_{i} \in \mathbb{H}^{(s)} \backslash K,
$$

which readily implies (4.32).

(B) We now use Theorem 4.3 and Corollary 4.8 to estimate the difference between a Taylor polynomial and a Chung-Yao interpolation polynomial of a same function. To simplify, we omit the index $s$ in the formulas. We have

$$
\begin{aligned}
& \mathbf{L}[\Theta ; f](x)-\mathbf{T}_{0}^{d-N}(f)(x)=\left[f(x)-\mathbf{T}_{0}^{d-N}(f)(x)\right]-[f(x)-\mathbf{L}[\Theta ; f](x)] \\
& =\sum_{K \in\left(\begin{array}{l}
\mathbb{H}_{d} d \\
N-1
\end{array}\right)} P_{K}^{[d]}(x)\left(\left[0, \ldots, 0, x \mid \mathbf{n}_{K}, \ldots, \mathbf{n}_{K}\right] f-\left[\Theta_{K}, x \mid \mathbf{n}_{K}, \ldots, \mathbf{n}_{K}\right] f\right) \\
& \quad+\sum_{i=N}^{d} \sum_{K \in\left(\begin{array}{c}
\mathbb{H}_{i-1} \\
N-1
\end{array}\right)} P_{K}^{[i-1]}(x) \int_{[0, \ldots, 0, x]} f^{(d-N+1)}(\cdot)\left(x^{d-i}, \vartheta_{K \cup \ell_{i}}, \mathbf{n}_{K}^{i-N}\right), \\
& \quad x \in B(0, R) .
\end{aligned}
$$


We call the terms in the above sum $S_{1}(x)$ and $S_{2}(x)$ and prove that, for every $x \in B(0, R)$, both of them tend to 0 as $s \rightarrow \infty$. This will achieve the proof. Simple convergence on a compact set of nonempty interior is sufficient since it implies convergence on any normed vector space topology on $\mathcal{P}^{d-N}\left(\mathbb{R}^{N}\right)$ (but the proof actually leads to uniform bounds indicated below).

(C) Since, in view of (4.32), the polynomials $P_{K}^{[d]}$ are bounded uniformly in $s$, that $S_{1}(x) \rightarrow 0$ for $x \in B(0, R)$ follows from

$$
\left|[\underbrace{0, \ldots, 0}_{d-N+1}, x \mid \underbrace{\mathbf{n}_{K}, \ldots, \mathbf{n}_{K}}_{d-N+1}] f-\left[\Theta_{K}, x \mid \mathbf{n}_{K}, \ldots, \mathbf{n}_{K}\right] f\right| \rightarrow 0,
$$

which is a consequence of the fact that the points of $\Theta=\Theta^{(s)}$ tend to 0 together with the continuity of the divided differences of $f$ as a function of the two groups of its arguments, see $\S 4.2$.

(D) As for the term $S_{2}(x)$, since the right-hand side goes to 0 as $s \rightarrow \infty$, the conclusion follows from the following estimate.

$$
\left|S_{2}(x)\right| \leqslant \frac{M}{(d-N+1) !} R^{d-N}\left(1+\frac{2}{\delta}\right)^{d-1}\|\Theta\|, \quad x \in B(0, R),
$$

where $\|\Theta\|=\left\|\Theta^{(s)}\right\|:=\max \{\|\vartheta\|: \vartheta \in \Theta\}$. To prove this, we observe that if $N \leqslant i \leqslant d$ and $K \in\left(\begin{array}{c}\mathbb{H}_{i-1} \\ N-1\end{array}\right)$, the bound (4.32) (in which $\mathbb{H}$ is replaced by $\mathbb{H}_{i-1}$ ) gives

$$
\left|P_{K}^{[i-1]}(x)\right| \leqslant\left(\frac{2 R}{\delta}\right)^{i-N}, \quad x \in B(0, R) .
$$

Moreover, for every $a \in B(0, R)$, using $\left\|\mathbf{n}_{K}\right\| \leqslant 1$, we have

$$
\left|f^{(d-N+1)}(a)\left(x^{d-i}, \vartheta_{K \cup \ell_{i}}, \mathbf{n}_{K}^{i-N}\right)\right| \leqslant M\|x\|^{d-i}\left\|\vartheta_{K \cup \ell_{i}}\right\|\left\|\mathbf{n}_{K}\right\|^{i-N} \leqslant M R^{d-i} \cdot\|\Theta\| .
$$

Hence, since $\operatorname{vol}\left(\Delta_{d-N+1}\right)=1 /(d-N+1)$ !, for $x \in B(0, R)$ we have

$$
\left|\int_{[0, \ldots, 0, x]} f^{(d-N+1)}(\cdot)\left(x^{d-i}, \vartheta_{K \cup \ell_{i}}, \mathbf{n}_{K}^{i-N}\right)\right| \leqslant \frac{M}{(d-N+1) !} R^{d-i}\|\Theta\| .
$$

Combining the above estimates, we obtain

$$
\begin{aligned}
\left|S_{2}(x)\right| & \leqslant \sum_{i=N}^{d}\left(\begin{array}{c}
i-1 \\
N-1
\end{array}\right)\left(\frac{2 R}{\delta}\right)^{i-N} \frac{M}{(d-N+1) !} R^{d-i}\|\Theta\| \\
& =\frac{M}{(d-N+1) !}\|\Theta\| R^{d-N} \sum_{i=N}^{d}\left(\begin{array}{c}
i-1 \\
i-N
\end{array}\right)\left(\frac{2}{\delta}\right)^{i-N} \\
& \leqslant \frac{M}{(d-N+1) !}\|\Theta\| R^{d-N} \sum_{j=0}^{d-1}\left(\begin{array}{c}
d-1 \\
j
\end{array}\right)\left(\frac{2}{\delta}\right)^{j} \\
& =\frac{M}{(d-N+1) !}\|\Theta\| R^{d-N}\left(1+\frac{2}{\delta}\right)^{d-1} .
\end{aligned}
$$

This concludes the proof of Theorem 3.1.

\subsection{An estimate on the error}

The proof actually yields a uniform bound on the error between Chung-Yao interpolation polynomials and the Taylor polynomial at the origin. It is shown in the following corollary. 
Corollary 4.9. We assume that the assumptions of Theorem 3.1 are satisfied. If $f \in$ $C^{d-N+2}(\Omega)$ then

$$
\begin{aligned}
& \max _{x \in B(0, R)}\left\|\mathbf{L}\left[\Theta^{(s)} ; f\right](x)-\mathbf{T}_{0}^{d-N}(f)(x)\right\| \\
& =O\left(\left\|\theta^{(s)}\right\| \cdot\left\{\max _{a \in B(0, R)}\left\|f^{(d-N+1)}(a)\right\|+\max _{a \in B(0, R)}\left\|f^{(d-N+2)}(a)\right\|\right\}\right),
\end{aligned}
$$

where the constant involved in the symbol $O$ does not depend on $f$.

Proof. We turn to the term $S_{1}(x)$ in the previous proof. For simplicity, we set $m=d-N+1$. Since $f \in C^{m+1}(\Omega)$, for all $K \in\left(\begin{array}{c}\mathbb{H}_{d} \\ N-1\end{array}\right)$, the mean value inequality gives

$$
\begin{aligned}
& \left|\left[0, \ldots, 0, x \mid \mathbf{n}_{K}, \ldots, \mathbf{n}_{K}\right] f-\left[\Theta_{K}, x \mid \mathbf{n}_{K}, \ldots, \mathbf{n}_{K}\right] f\right| \\
& \quad=\left|\int_{\Delta_{m}}\left\{f^{(m)}\left(x+\sum_{j=1}^{m}(0-x) \xi_{j}\right)\left(\mathbf{n}_{K}^{m}\right)-f^{(m)}\left(x+\sum_{j=1}^{m}\left(\theta_{K j}-x\right) \xi_{j}\right)\left(\mathbf{n}_{K}^{m}\right)\right\} d \xi\right| \\
& \quad \leqslant \int_{\Delta_{m}} \max _{B(0, R)}\left\|f^{(m+1)}\right\|\left\|\sum_{j=1}^{m} \theta_{K j} \xi_{j}\right\|\left\|\mathbf{n}_{K}\right\|^{m} d \xi \leqslant \frac{1}{m !} \max _{B(0, R)}\left\|f^{(m+1)}\right\|\|\Theta\|,
\end{aligned}
$$

where $\Theta_{K}=\left\{\theta_{K j}: j=1, \ldots, m\right\}$. Using (4.36) and (4.45) in (4.34), we finally get

$$
\begin{aligned}
& \max _{x \in B(0, R)}\left\|\mathbf{L}[\Theta, f](x)-\mathbf{T}_{0}^{d-N}(f)(x)\right\| \\
& \leqslant\left(\begin{array}{c}
d \\
N-1
\end{array}\right)\left(\frac{2 R}{\delta}\right)^{d-N+1} \frac{1}{(d-N+1) !} \max _{B(0, R)}\left\|f^{(d-N+2)}\right\|\|\Theta\| \\
&+\frac{1}{(d-N+1) !} \max _{B(0, R)}\left\|f^{(d-N+1)}\right\| R^{d-N}\left(1+\frac{2}{\delta}\right)^{d-1}\|\Theta\| \\
&=\left(M_{1} \max _{B(0, R)}\left\|f^{(d-N+1)}\right\|+M_{2} \max _{B(0, R)}\left\|f^{(d-N+2)}\right\|\right)\|\Theta\| .
\end{aligned}
$$

Acknowledgements. We would like to thank the referee for pointing out several useful references. The work of Phung Van Manh was supported by a $\mathrm{PhD}$ fellowship from the Vietnamese government.

\section{References}

1. T. Bloom and J.-P. Calvi, 'A continuity property of multivariate Lagrange interpolation', Math. Comp. 66 (1997) no. 220, 1561-1577.

2. J. Carnicer, M. Gasca and T. Sauer, 'Aitken-Neville sets, principal lattices and divided differences', J. Approx. Theory 156 (2009) no. 2, 154-172.

3. K. C. Chung and T. H. YaO, 'On lattices admitting unique Lagrange interpolations', SIAM J. Numer. Anal. 14 (1977) no. 4, 735-743.

4. C. DE Boor, 'The error in polynomial tensor-product, and Chung-Yao interpolation', Surface fitting and multiresolution methods Chamonix-Mont-Blanc, 1996 (Vanderbilt University Press, Nashville, TN, 1997) $35-50$.

5. C. DE Boor and B. Shekhtman, 'On the pointwise limits of bivariate Lagrange projectors', Linear Algebra Appl. 429 (2008) no. 1, 311-325.

6. M. GASCA and T. SAUER, 'On bivariate Hermite interpolation with minimal degree polynomials', SIAM J. Numer. Anal. 37 (2000) no. 3, 772-798.

7. C. A. Micchelli, 'A constructive approach to Kergin interpolation in $\mathbf{R}^{k}$ : multivariate $B$-splines and Lagrange interpolation', Rocky Mountain J. Math. 10 (1980) no. 3, 485-497.

8. T. SAUER, 'Polynomial interpolation in several variables: lattices, differences, and ideals', Topics in multivariate approximation and interpolation, Studies in Computational Mathematics 12 (Elsevier, Amsterdam, 2006) 191-230.

9. T. SAuER and Y. Xu, 'Regular points for Lagrange interpolation on the unit disk', Numer. Algorithms 12 (1996) no. 3-4, 287-296. 
10. B. Shekhtman, 'On a conjecture of Carl de Boor regarding the limits of Lagrange interpolants', Constr. Approx. 24 (2006) 365-370, doi:10.1007/s00365-006-0634-7.

11. B. Shekhtman, 'On the limits of Lagrange projectors', Constr. Approx. 29 (2009) 293-301, doi:10.1007/s00365-008-9016-0.

J.-P. Calvi

Institut de Mathématiques

Université de Toulouse III and CNRS

(UMR 5219)

31062, Toulouse Cedex 9, France

jean-paul.calvi@math.univ-toulouse.fr
V. M. Phung

Department of Mathematics

Hanoi University of Education

136 Xuan Thuy Street

Caugiay, Hanoi, Vietnam

manhlth@gmail.com 